\title{
Vom Standard zur Leitlinie: Bewertungszusammenhänge im Medizin- und Gesundheitsrecht
}

- Leitlinie und Standard, EbM und HTA, Pflicht und Zweckmäßigkeit -

\section{Einleitung}

Im Zentrum dieses Beitrags stehen Leitlinien. Leitlinien können als klinische bzw. ärztliche allgemeine medizinische Handlungsstrategien für die individuelle Behandlung von Krankheiten oder als Versorgungsleitlinien allgemeine Handlungsstrategien für Leistungen der Gesundheitsversorgung entwickeln. Für den ersten Typus der klinischen Leitlinien ist die ärztliche Profession zuständig, für den zweiten Typus der Versorgungsleitlinien sind multidisziplinäre und multiprofessionelle Kompetenzen erforderlich. Begreift man Leitlinien als ein Instrument der Umsetzung von Handlungsstrategien im Medizin- und im Gesundheitssystem, dann beziehen sich klinische Leitlinien auf die Methode der evidenzbasierten Medizin, während Versorgungsleitlinien sich auf die Methode des Health Technology Assessment (HTA) gründen. Das Medizinrecht hat es mit den klinischen, das Gesundheitsrecht mit den Versorgungsleitlinien zu tun. Medizinrecht ist individuell behandlungsbezogen, Gesundheitsrecht allgemein leistungs- und versorgungssystembezogen. Der medizinische Standard orientiert sich am Maß der Evidenz-basierten Medizin (EbM) und ist die medizinrechtlich geschuldete Sorgfalt; der anerkannte Stand der medizinischen Erkenntnisse, die Zweckmäßigkeit und die Wirtschaftlichkeit sind die gesundheitsrechtlichen Anknüpfungspunkte der Rezeption für EbM und HTA.

Der medizinische Standard ist ein Zentrum aller medizin- und gesundheitsrechtlichen Überlegungen und das $\mathrm{Ma} \beta$, an dem sich die Leitlinien gleich welcher Couleur zu orientieren haben. Nur dem Standard entsprechende und nach ihm aktualisierte Leitlinien können eine medizinische und demzufolge eine rechtliche Verbindlichkeit begründen. Es scheint deshalb in jeder Hinsicht der Satz zu gelten:

"Die Leitlinie ist medizinisch verbindlich, wenn sie dem Standard entspricht und ist rechtlich verbindlich, weil sie dem Standard entspricht. ${ }^{1}$

Ausgangspunkt für jedwede Verbindlichkeitswirkung ist danach üblicherweise der medizinische Standard als Synonym für wissenschaftliche und praktische Lernprozesse in der Medizin sowie in der Folge im Medizin- und Gesundheitsrecht.

Überblicksartig sei das folgende Schaubild schematisiert:

1 Hart, Leitlinien und Haftungsrecht - Inkorporation, Rezeption und Wissensbasis in Wissenschaft und Praxis, in: Hart (Hrsg.), Klinische Leitlinien und Recht, 2005, S. 81 ff., bes. $96 \mathrm{f}$. 
Schaubild 1

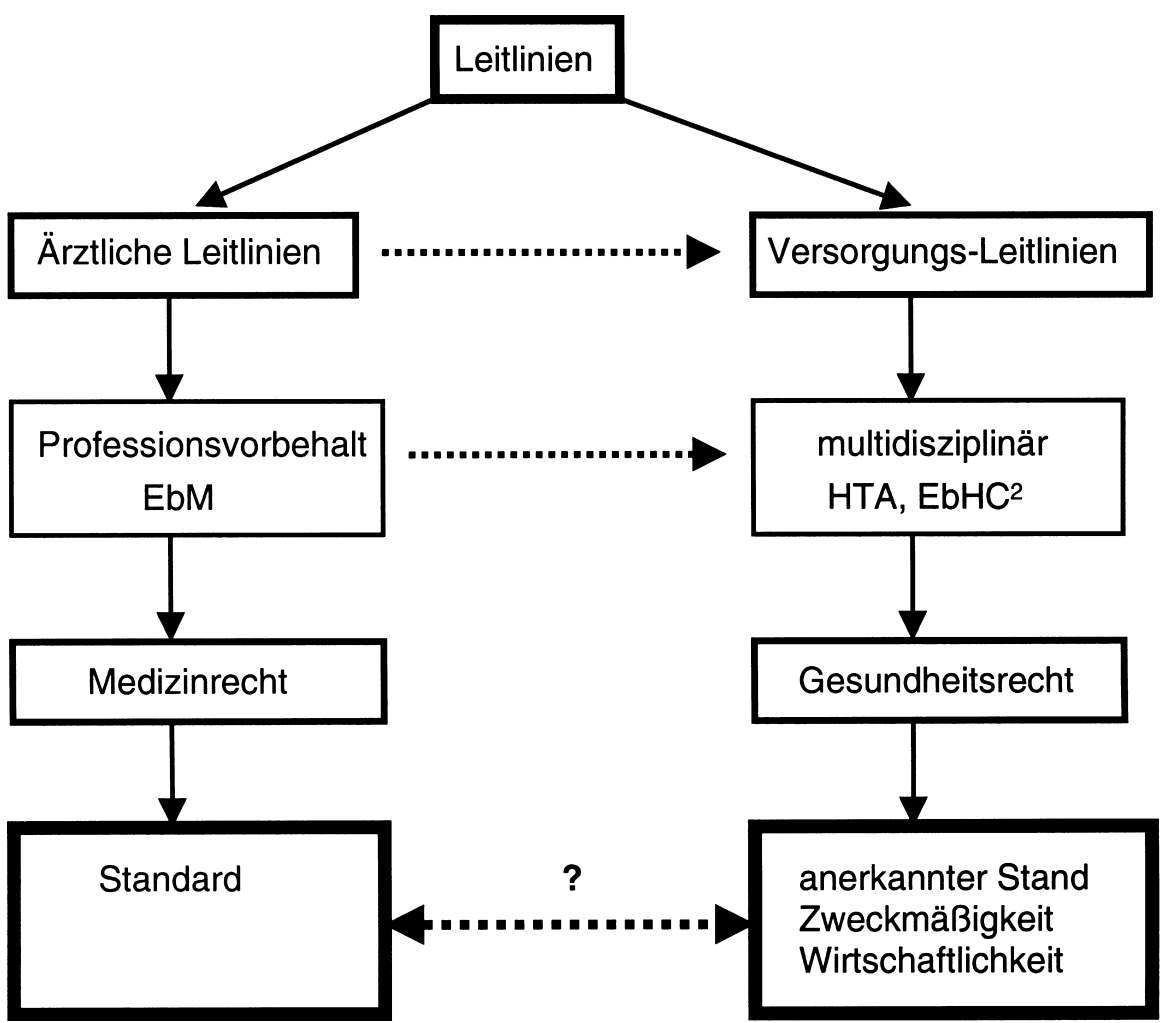

Die den vorangehenden Ausführungen und dem Schaubild zugrunde liegenden Zusammenhänge und Beziehungen sind außerordentlich voraussetzungsvoll und nicht nur dort zweifelhaft, wo das Fragezeichen platziert ist. Man kann sie mit guten Gründen in Frage stellen und nicht mehr den Begriff des Standards, sondern den der Leitlinie in das Zentrum der Überlegungen rücken.

Deshalb lautet die hier tendenziell vertretene und in ihren Konsequenzen verfolgte These:

Die Leitlinie bestimmt den Standard, je weiter der Prozess der Harmonisierung der Verfahren und Kriterien der Leitlinienerstellung voranschreitet und die Praxis dies implementiert. Die Leitlinie stellt nicht mehr nur den Standard fest (»Deklaration«), sondern sie setzt ihn fest (»Konstitution«).

2 Evidence based Health Care. 
Im Zentrum der Normbildung stehen insofern der Prozess der Leitlinienerstellung und seine verfahrensmäßigen und sachlichen Qualitätskriterien, die einzuhalten sind. Macht man diesen Prozess zum Kern des Normbildungsprozesses, dann handelt es sich um eine grundlegende Umorientierung der Konsens- und Akzeptanzbildungs-, aber auch Lernprozesse in der Medizin, die nicht mehr eher naturwüchsig, sondern systematisch strukturiert zu verlaufen beginnen und in einer neuen Weise regulatorisch verfasst sind bzw. werden. Das medizin- wie gesundheitsrechtliche regulatorische System wird sich darauf einzustellen haben.

Diese Herangehensweise müsste man folgendermaßen schematisieren:

Schaubild 2

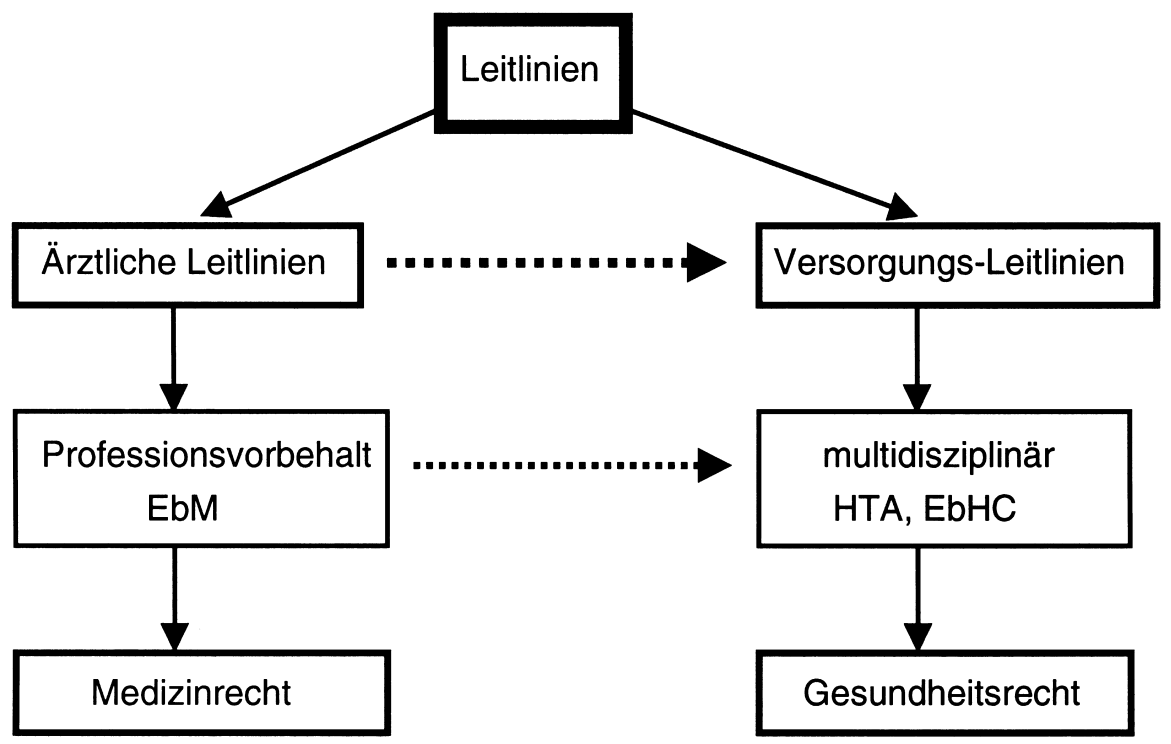

In beiden Schaubildern erscheinen die dritte Zeichenebene und die Quer-Verbindungspfeile der zweiten und dritten Zeichenebene identisch. Die dritte Zeichenebene ist das Vermittlungsglied zwischen beiden Lern- oder Handlungsmodellen.

Der folgende Text versucht diese Zusammenhänge zu erläutern und zu begründen und dadurch einen Beitrag zum Verhältnis und zur Harmonisierung von Medizinrecht und Gesundheitsrecht zu leisten. Er geht von einem gegenüber den bisherigen Beiträgen des Verfassers zu diesem Thema »radikalisierten« Ansatz aus, entwickelt seine wesentlichen Konsequenzen und stellt ihn zur Diskussion.

Zunächst wird ein kurzer Überblick über die Quantität, Qualität der und das Verfahren des Zustandekommens von Leitlinien (Empirie) gegeben und die Unterscheidung der Leitlinientypen vorgestellt (II). Dem folgt eine knappe Präsentation von EbM und HTA, für die die Leitlinien in doppelter Weise Umsetzungs- oder Vollzugsinstrumente darstellen (III). Der anschließende Abschnitt gilt dem Verhältnis von Leitlinien und Standard im Medizinrecht (IV) und im Gesundheitsrecht (V). 


\section{Definitionen und Leitlinienempirie}

Am Beginn seien die nahezu allgemein akzeptierten Definitionen der zentralen Begriffe dargestellt und ihre wechselbezüglichen Relationen erläutert. ${ }^{3}$

\section{Definitionen}

»Leitlinien sind systematisch entwickelte Entscheidungshilfen über die angemessene ärztliche Vorgehensweise bei speziellen gesundheitlichen Problemen. ... Leitlinien sind wissenschaftlich begründete und praxisorientierte Handlungsempfehlungen. ... Leitlinien sind Orientierungshilfen im Sinne von »Handlungs- und Entscheidungskorridoren«, von denen in begründeten Fällen abgewichen werden kann oder sogar muß. ${ }^{4}$

Ärztliche Leitlinien haben wissenschaftliche, praktische, informatorische und regulatorische (umsetzende) Funktionen. Wissenschaftlich sollen sie den Stand der Erkenntnisse zum Zeitpunkt ihres Erlasses (»state of the art«) repräsentieren; praktisch führen sie die ärztliche Tätigkeit an die wissenschaftlichen Erkenntnisse heran, indem sie die Ärzteschaft darüber informieren und auf diese Weise zur Durchsetzung des wissenschaftlich erreichten Standes in der Praxis beitragen (Implementierung). Erfüllen die Leitlinien diese Funktionen, tragen sie zur Qualitätsverbesserung ärztlicher Behandlungen und zum Patientenschutz bei, indem sie den festgestellten medizinischen Standard, der sich aus wissenschaftlicher Erkenntnis, praktischer Erfahrung und professioneller Akzeptanz (»Konsens«) zusammensetzt, umsetzen. ${ }^{5}$ Leitlinien dienen deshalb auch als Fehlerpräventions- und Fehleridentifikationsinstrumente. ${ }^{6}$

Versorgungsleitlinien ${ }^{7}$ sind multidisziplinäre Instrumente der Regulierung u. a. des Nutzens, der Effektivität und der Effizienz von medizinischen Behandlungen im Rahmen eines Gesundheitssystems. Sie sollten auf ärztlichen Leitlinien beruhen. Ihr Gesundheitssystembezug ist durch zwei Momente gekennzeichnet: Versorgung ist eine kollektive Leistung des sozialen Sicherungssystems und sie ist als Systemleistung abhängig von ihrer planbar effizienten Erbringung. Der Gesundheitssystembezug bringt

3 Überschneidungen mit einem jüngsten Text lassen sich in diesem Teil nicht vermeiden: Hart, Leitlinien und Haftungsrecht, in: Hart (Hrsg.), Klinische Leitlinien und Recht, S. 81 ff., bes. 88 ff.; im Sommer 2005 wird der Abschlussbericht eines von der VolkswagenStiftung finanzierten Forschungsprojektes zu ärztlichen Leitlinien erscheinen, Hart (Hrsg.), Ärztliche Leitlinien im Medizin- und Gesundheitsrecht - Recht und Empirie professioneller Normbildung, Baden-Baden.

4 Beurteilungskriterien für Leitlinien in der medizinischen Versorgung, Beschlüsse der Vorstände von Bundesärztekammer und Kassenärztlicher Bundesvereinigung v. Juni 1997, DÄBl. 1997, (Heft 33) A-2154-2155; siehe insgesamt jüngst Ollenschläger/Kirchner/ Sänger/Thomeczek/Jonitz/Gramisch, Qualität und Akzeptanz medizinischer Leitlinien in Deutschland - Bestandsaufnahme Mai 2004, in: Hart (Hrsg.), Klinische Leitlinien und Recht, 2005, S. $17 \mathrm{ff}$.

5 Dazu Ollenschläger/Kirchner/Fiene, Leitlinien in der Medizin - scheitern sie an der praktischen Umsetzung?, Internist 2001·42:473-483.

6 Hansis/Hansis, Der ärztliche Behandlungsfehler - Verbessern statt streiten, Landsberg: ecomed, 1999; Becker-Schwarze/Hart, Die juristische Sicht: Konflikt zwischen Haftungsrecht und Incident Reporting?, GesR 1/2005, 1-5.

7 Hart, Qualitätssicherung durch Leitlinien, VSSR 2002, 265-297, bes. 268. 
es darüber hinaus mit sich, dass ihre Verbindlichkeit nicht mehr einem innerprofessionellen Konsens geschuldet ist, wie das prinzipiell für die ärztliche Leitlinie gilt, sondern dass es sich um eine multidisziplinäre und multiprofessionelle Veranstaltung handelt, deren Normcharakter durch rechtliche »Verleihung « zustande kommt. Versorgungsleitlinien sind deshalb in gewisser Weise ein $» Z w i t t e r ~ «{ }^{8}$ Materiell verbinden sie die medizinische Bewertung von Behandlungen mit ihrer gesundheitswissenschaftlichen und gesundheitsökonomischen Bewertung. Formell können sie den Charakter von sozialrechtlichen Richtlinien annehmen, wenn sie entsprechend rechtlich rezipiert werden. ${ }^{9}$

Dem Standard entspricht ein ärztliches Handeln, das nach medizinisch-wissenschaftlicher Erkenntnis und/oder ärztlicher Erfahrung innerhalb der Profession akzeptiert ist. Diese Definition des medizinischen Standards entspricht im Wesentlichen den medizinliterarischen ${ }^{10}$ Stellungnahmen und denen der ärztlichen Selbstverwaltungsorgane ${ }^{11}$ und ist ebenso in der juristischen Literatur ${ }^{12}$ präsent. Der medizinische Standard setzt sich also aus den drei Elementen

- wissenschaftliche Erkenntnis,

- praktische Erfahrung und

- Akzeptanz in der Profession

8 Der hier gewählte Begriff der Versorgungsleitlinie hat nichts mit dem von der Bundesärztekammer betriebenen Nationalen Programm für Versorgungsleitlinien bei der Bundesärztekammer (ZaeFQ (2002) 96:Suppl. II, 1-24) zu tun, das durch Diskussionen zu § 137 f. SGB V angestoßen wurde und sozusagen die »abgespeckte« Variante der qualitativ höchstwertigen Leitlinien darstellt; siehe insgesamt http://www.leitlinien.de/versorgungsleitlinien/index/view; dazu auch Ollenschläger u. a., Qualität und Akzeptanz, S. 17 ff., bes. $31 \mathrm{ff}$.

9 Dazu insgesamt Mengel, Sozialrechtliche Rezeption ärztlicher Leitlinien, 2004, S. 109 ff.

10 Vgl. zu diesem Begriff in der medizinischen Literatur Buchborn, Verbindlichkeit medizinisch wissenschaftlicher Aussagen in der ärztlichen Praxis, in: Deutsch/Kleinsorge/Scheler (Hrsg.), Verbindlichkeit der medizinisch-diagnostischen und therapeutischen Aussage, 1983, S. 107, 110 ff.; ders., Der Ärztliche Standard, DÄB1. 1993, C-1322 ff.; ders., Ärztlicher Standard: Begriff - Entwicklung - Anwendung, MedR 1993, 328 ff.; Carstensen, Vom Heilversuch zum medizinischen Standard, DÄBl. 1989, B-1736; C-1531.

11 Vgl. Qualitätssicherung und Qualitätsverbesserung in der Medizin - Grundlagen einer bedarfsgerechten Gesundheitsversorgung, Gemeinsame Bestandsaufnahme der Bundesärztekammer und der Kassenärztlichen Bundesvereinigung über die Aktivitäten der Spitzenorganisationen der ärztlichen Selbstverwaltung auf dem Gebiet der Qualitätssicherung in der Medizin, 1996; Curriculum Qualitätssicherung, Teil 1: Ärztliches Qualitätsmanagement, Bundesärztekammer, Kassenärztliche Bundesvereinigung, Arbeitsgemeinschaft der Wissenschaftlichen Medizinischen Fachgesellschaften (Hrsg.), Texte und Materialien der Bundesärztekammer zur Fortbildung und Weiterbildung, Bd. 10, 1996, dort insbes. die Beiträge von Ollenschläger/Thomeczek, Ärztliche Leitlinien - Definitionen, Ziele, Implementierung, S. 177 ff. und Schirmer, Rechtliche Anmerkungen zum Problem der Empfehlungen, Leitlinien, Richtlinien und Standards in der Medizin, S. $187 \mathrm{ff}$.

12 Vgl. Hart, Ärztliche Leitlinien - Definitionen, Funktionen, rechtliche Bewertungen Gleichzeitig ein Beitrag zum medizinischen und rechtlichen Standardbegriff; MedR 1998, 8 ff. mit weiteren Nachweisen; ders., Leitlinien und Haftungsrecht, in: Hart (Hrsg.), Klinische Leitlinien und Recht, S. 81 ff., bes. 88 ff.; Kern, Führt die Vorgabe von Standards, Leitlinien und ökonomischen Begrenzungen auch zu einer Veränderung des zivilrechtlichen Haftungsmaßstabes?, ZaeFQ (2004) 98:222-226; Schreiber, Rechtliche Gesichtspunkte, in: Nagel/Fuchs (Hrsg.), Leitlinien und Standards im Gesundheitswesen, 1997, S. 167 ff. 
zusammen. Alle drei Definitionselemente bedürfen der Präzisierung und Interpretation, weil festgelegt werden muss, welches Maß und welcher Grad an wissenschaftlicher Erkenntnis erforderlich sind, welches Maß und welcher Grad an praktischer ärztlicher Erfahrung erforderlich sind, welches Maß an Akzeptanz in der Profession (von Konsens bis zur Mehrheit der wissenschaftlichen Experten und Praktiker) zu verlangen ist. Dies ist eine wichtige Funktion von EbM. ${ }^{13}$

Das folgende Schema stellt den üblicherweise angenommenen Zusammenhang dar.

\section{Schaubild 3}

\section{Standard}
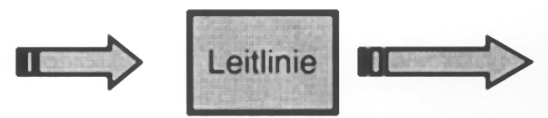

Versorgungsleitlinie

Der medizinische Standard ist das Fundament für die ärztliche Leitlinie und diese wiederum die Basis der Versorgungsleitlinie, die aber nicht nur medizinischen Beurteilungen, sondern zusätzlich gesundheitswissenschaftlichen und ökonomischen Bewertungen folgt. Dies ist eine wichtige Funktion von HTA.

Das Modell geht wie im Schaubild 1 davon aus, dass der bestehende Standard weder durch die Leitlinie noch die Versorgungsleitlinie geändert werden darf - also auch Wirtschaftlichkeitserwägungen nur auf ihm aufbauen, ihn aber nicht deformieren (dürfen, sollen).

\section{Empirie}

Leitlinien werden aufgestellt von internationalen und nationalen Ärzteorganisationen, insbesondere von medizinischen Fachgesellschaften, aber auch von regionalen (z. B. Ärztekammern, Fachorganisationen in den Ländern) und lokalen (bis hin zu Krankenhausabteilungen und Praxisnetzen) Institutionen. Die Zahl der Leitlinien der deutschen $A W M F$, also der Arbeitsgemeinschaft der wissenschaftlichen medizinischen Fachgesellschaften, hat inzwischen 950 überschritten. ${ }^{14}$

Der Entwicklungsprozess der Erstellung von ärztlichen Leitlinien setzte international etwa Ende der 70er Jahre des letzten Jahrhunderts, in Deutschland erst Mitte der 90er Jahre ein. Die Leitlinien-Bewegung ist eng mit der anglo-amerikanischen EbMBewegung verbunden und mit der Etablierung dieses Ansatzes in Deutschland in den letzten 10 Jahren hat sich auch die Qualität der nationalen Leitlinien erheblich verän-

13 Siehe Raspe, Von der Evidenz zur Empfehlung - Zum Verhältnis von wissenschaftlicher Erkenntnis und Erfahrung, in: Hart (Hrsg.), Klinische Leitlinien und Recht, 2005, S. 63 ff.

14 Siehe Encke/Reinauer/Lorenz, Weiterentwicklung der Qualität der Gesundheitsversorgung unter Nutzung wissenschaftlich basierter Leitlinien, Leitlinien-Publikationen, http:// www.leitlinien.net/wb-ll.htm; sehr guter Überblick bei Kopp/Encke/Hartig/Müller/Lorenz, Zur Empirie hochwertiger Leitlinien im System der Arbeitsgemeinschaft Wissenschaftlicher Medizinischer Fachgesellschaften (AWMF): Gibt es sie und wie viele?, in: Hart (Hrsg.), Klinische Leitlinien und Recht, 2005, S. 41 ff. 
dert. Während anfangs (bis etwa 1998) der naturwüchsige Konsens der Peers im Vordergrund der Leitlinienproduktion stand, ist es nunmehr die an Evidenzkriterien und formalisierten Verfahren der Konsensfindung orientierte Methodik, die die Qualität der Leitlinie definiert.

Der Prozess der Harmonisierung der methodischen Anforderungen an nationale Leitlinien zwischen Wissenschaft, Selbstverwaltung und Praxis ist inzwischen weit vorangeschritten ${ }^{15}$ und in einem gemeinsamen Konsensuspapier publiziert worden. ${ }^{16}$ Darin wird der »evidenz-basierten Konsensus-Leitlinie « die höchste wissenschaftliche und gesundheitspolitische Legitimation zugesprochen. Dieses Konsens-Papier ${ }^{17}$ betont zwei elementare Anforderungen an die Leitlinie:

evidenz-basiert, $d$. $h$. die Leitlinie hat sich an der bestmöglichen erreichbaren Evidenz zu orientieren und

Konsensus: es bedarf einer professionellen Akzeptanzbewertung in einem geordneten Verfahren der Konsensfindung.

Und: die »evidenz-basierte Konsensus-Leitlinie« kann sowohl ärztliche Leitlinie wie Versorgungsleitlinie sein. Vorausgesetzt wird die Transparenz der Bewertungen: medizinische und Effizienz-Bewertungen sind auseinander $\mathrm{zu}$ halten und offen $\mathrm{zu}$ legen.

In eine ähnliche Richtung geht die am 10. Oktober 2001 verabschiedete Empfehlung des Europarates zu Leitlinien. ${ }^{18}$ Es existiert inzwischen ein Internationales LeitlinienNetzwerk (Guidelines International Network) ${ }^{19}$, in dem wichtige nationale LeitlinienOrganisationen zusammengeschlossen sind (aus Deutschland u. a. ÄZQ, AWMF). Das Netz dient in erster Linie der gegenseitigen Information über die internationale Leitlinienentwicklung und Methodik ihrer Erstellung. ${ }^{20}$ International und in Staaten mit einer Leitlinien-Tradition, also z. B. UK, Kanada, existieren EbM-Leitlinien, die den

15 Ollenschläger u. a., Qualität und Akzeptanz, S. 17 ff., bes. $20 \mathrm{ff}$.

16 Die Einigung von Wissenschaft, Selbstverwaltung und Praxis auf evidenz-basierte Konsensus-Leitlinien, Helou/Lorenz/Ollenschläger/Reinauer/Schwartz, Methodische Standards der Entwicklung evidenz-basierter Leitlinien in Deutschland - Konsens zwischen Wissenschaft, Selbstverwaltung und Praxis, ZaeFQ 94 (2000), 330-339.

17 Siehe dazu Reinauer, Evidenz-basierte Medizin und ärztliche Leitlinien, Q-med 2001, $6 \mathrm{ff}$.

18 Recommendation $\operatorname{Rec}(2001) 13$ of the Committee of Ministers to member states on developing a methodology for drawing up guidelines on best medical practices (Adopted by the Committee of Ministers on 10 October 2001 at the $768^{\text {th }}$ meeting of the Ministers' Deputies); veröffentlicht in deutscher Übersetzung in ZaeFQ 96 (2002) Suppl. III,1-60.

19 Siehe http://www.g-i-n.net; Teil 5 dieses Berichts »Rechtsvergleichung und Internationales«, E. I., S. X.

20 Dazu Burgers/Grol/Klazinga/van der Bij/Mäkelä/Zaat und The AGREE Collaborative Group, Internationaler Vergleich von 19 Leitlinien-Programmen - Eine Übersicht der AGREE Collaboration, ZaeFQ (2003) 97:81-88; Informationen über die Arbeit von GIN werden regelmäßig in den Ausgaben der ZaeFQ (Zeitschrift für ärztliche Fortbildung und Qualität im Gesundheitswesen) unter der Rubrik »Aus dem Guidelines International Network« veröffentlicht. 
Anforderungen der »evidenz-basierten Konsensus-Leitlinien « entsprechen und in vergleichbaren Verfahren zustande gekommen sind. ${ }^{21}$

Die nationalen »evidenz-basierten Konsensus-Leitlinien « gibt es bisher nur in geringer, aber ständig steigender Zahl. Ihnen gehört die Zukunft. Viele der heute in Deutschland existierenden nationalen Leitlinien erreichen das verabredete Ziel nur bedingt. Vielen veröffentlichten »älteren« (ärztlichen) Leitlinien der AWMF wurde eine unzureichende insbesondere methodische Qualität attestiert. ${ }^{22}$

Die AWMF ${ }^{23}$ unterscheidet drei Stufen von Leitlinien, wobei die letzte Stufe die eigentliche Zielsetzung der Leitlinienentwicklung ist und möglichst am Ende eines jeden Leitlinienentwicklungsprozesses stehen sollte:

\section{S1-Leitlinien:}

Eine repräsentativ zusammengesetzte Expertengruppe der Wissenschaftlichen Medizinischen Fachgesellschaft erarbeitet im informellen Konsens eine Leitlinie, die vom Vorstand der Fachgesellschaft verabschiedet wird.

\section{S2-Leitlinien:}

Vorhandene Leitlinien der Stufe 1 werden in einem der bewährten formalen Konsensusverfahren beraten und als Leitlinien der Stufe 2 verabschiedet (nominaler Gruppenprozess, Konsensuskonferenz, Delphikonferenz), d. h. Diskussion der Evidenz für die verabschiedeten Statements in einem formalen Konsensverfahren.

\section{S3- Leitlinien:}

Die Leitlinienentwicklung der 2. Stufe wird auf folgende 5 Komponenten erweitert: Logik, Konsensus, Evidence based Medicine, Entscheidungsanalyse, outcome-Analyse, d. h. systematische Analyse der Evidenz .... in einem formalen Konsensverfahren.

In einer jüngsten Veröffentlichung geht die AWMF selbst davon aus, dass von den rund 950 Leitlinien der Wissenschaftlichen Fachgesellschaften etwa 189 der eigenen Klassifizierung S2 und S3 entsprechen, also als »evidenz-basierte Konsensus-Leitlinien« anzusehen seien; 753 sind S1-Leitlinien. Nach unserer Bewertung sind nur die

21 Auch dazu Burgers/Grol/Klazinga/van der Bij/Mäkelä/Zaat und The AGREE Collaborative Group, Internationaler Vergleich von 19 Leitlinien-Programmen - Eine Übersicht der AGREE Collaboration, ZaeFQ (2003) 97:81-88.

22 Helou/Perleth/Bitzer/Dörning/Schwartz, Qualität ärztlicher Leitlinien in Deutschland Ergebnisse einer systematischen Untersuchung deutscher Leitlinien im Internet, ZaeFQ 92 (1998), 421-428, die für die Mehrzahl der Leitlinien die Nichtübereinstimmung mit international anerkannten Qualitätskriterien konstatiert; siehe auch Helou/Ollenschläger, Ziele, Möglichkeiten und Grenzen der Qualitätsbewertung von Leitlinien, ZaeFQ 92 (1998), 361-365.

23 Siehe AWMF online, Leitlinien für Diagnostik und Therapie, Enke/Lorenz/Reinauer, Weiterentwicklung der Qualität der Gesundheitsversorgung unter Nutzung wissenschaftlich basierter Leitlinien, Stand: 27.8.2001; http://www.leitlinien.net/wb-ll.htm und ebendort 4. Rundbrief: Lorenz, »Leitlinien der AWMF«: neue Strategie (März 2001); Kopp/Encke/ Hartig/Müller/Lorenz, Zur Empirie hochwertiger Leitlinien, in: Hart (Hrsg.), Klinische Leitlinien, S. $41 \mathrm{ff}$. 
bisher $27^{24}$ S3-Leitlinien so zu qualifizieren. Betrachtet man diesen Leitlinien-Entwicklungsprozess seit Mitte der 90er Jahre, so ist er einerseits durch die Harmonisierung von Kriterien und Verfahren und andererseits durch die stetige Zunahme hochwertiger Leitlinien aufgrund einer sich breit durchsetzenden Evidenz-Basierung gekennzeichnet. Uneingeschränkte Anerkennung finden heute in der ärztlichen Profession im Wesentlichen nur noch S3-Leitlinien. Diese Aussage bezieht sich insbesondere auf die wissenschaftliche Medizin (Fachgesellschaften), während in der »praktischen Medizin« (Berufsverbände) die »Leitlinien-Bewegung« häufig noch vor Barriere- und Akzeptanzproblemen steht. ${ }^{25}$

\section{Befunde}

Die Qualität der S3-Leitlinie lässt die Aussage zu, dass sie im Zeitpunkt ihrer Verabschiedung den Standard feststellt bzw. ihm entspricht. Von diesem Zeitpunkt ab können aufgrund der Dynamik der medizinischen Entwicklung Leitlinie und Standard auseinanderfallen und die Leitlinie kann hinter dem Standard zurückbleiben. Die gesamte Leitlinienliteratur und -praxis gehen daher von der Notwendigkeit der Evaluation und aktualisierenden Anpassung aus. ${ }^{26}$ Je systematischer diesem Postulat gefolgt wird, desto weniger werden S3-Leitlinie und der medizinische Standard auseinanderfallen.

Im Vergleich zu diesem seriositätsindizierten Verfahren der Leitlinienerstellung und seinen Qualitätsanforderungen fallen alle anderen in Deutschland praktizierten Verfahren der Standardfeststellung prozedural und qualitativ zurück. Weder die Verfahren bei den Gutachterkommissionen und Schlichtungsstellen noch die Heranziehung von medizinischen Sachverständigen in Gerichtsverfahren, weder die Lehrbuchliteratur noch die Arbeit der Berufsverbände oder Qualitätszirkel entsprechen den Anforderungen der »evidenz-orientierten Konsensus-Leitlinie « und sind deshalb zur Standardfeststellung erheblich weniger geeignet und weniger verlässlich.

Das Verfahren und die Kriterien der Erstellung dieser Leitlinien gewährleisten wie kein anderes Verfahren die Verwertung der vorhandenen bestmöglichen Evidenz in einem qualifizierten Konsensbildungsprozess unter Experten der beteiligten Disziplinen. Alle anderen Verfahren der Standardfeststellung sind diesem Verfahren unterlegen.

Diese Erkenntnis macht aus dem dreistufigen ein zweistufiges Modell:

24 9. Rundbrief vom September 2003: Ina Kopp, Wolfgang Müller, Wilfried Lorenz, Leitlinien im System der AWMF: aktueller Stand und Perspektiven.

25 Auch dazu 9. Rundbrief vom September 2003: Ina Kopp, Wolfgang Müller, Wilfried Lorenz, Leitlinien im System der AWMF: aktueller Stand und Perspektiven: Barrierenanalyse; siehe auch DÄBl online v. 15.2.2005 »Berufserfahrung kontra Leitlinien: Gründe für die Fortbildungsresistenz älterer Kollegen«, wo über US-amerikanische Studien berichtet wird, dass ältere Mediziner neue Therapien und Leitlinien in der Praxis seltener umsetzen (Annals of Internal Medicine 2005; 142:260-273).

26 Vgl. AWMF, Qualität von Leitlinien: Planmäßige Überprüfung; www.leitlinien.net Qualitätskriterien für Leitlinien; Kopp/Encke/Lorenz, Leitlinien als Instrument der Qualitätssicherung in der Medizin - Das Leitlinienprogramm der Arbeitsgemeinschaft Wissenschaftlicher Medizinischer Fachgesellschaften (AWMF), Bundesgesundheitsblatt Gesundheitsforschung - Gesundheitsschutz 45 (2002), 223-233. 
Schaubild 4
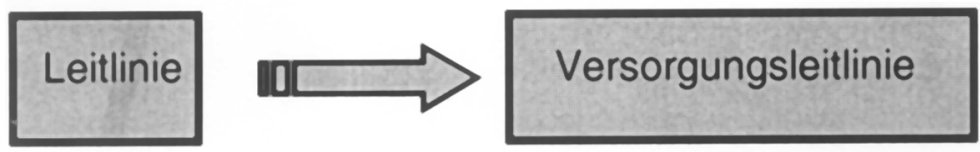

Daraus ergibt sich die Konsequenz:

Die S3-Leitlinie setzt den Standard fest; sie ist der Standard.

Je weiter der Prozess der Qualitätssteigerung der Leitlinien fortschreitet, desto eher geht die Funktion der »Standardfeststellung « auf die Leitlinien als Standardfestsetzung über. Die hochwertige Leitlinie wird zum Zentrum der Normbildung in der Medizin. Selbstverständlich steht diese Aussage unter dem Vorbehalt ordnungsgemäßer Evaluierung und Aktualisierung der Leitlinie.

Im folgenden Abschnitt soll diese These zusätzlich begründet und in den Zusammenhang der wissenschaftlichen Legitimation von Leitlinien in der modernen Medizin gestellt werden. EbM und HTA stehen dabei im Mittelpunkt der methodischen Fortentwicklung der Voraussetzungen und Verfahren medizinischer Normbildung. Die weiteren Abschnitte sind der medizin- und gesundheitsrechtlichen Beurteilung gewidmet.

\section{Leitlinien als Instrumente für EbM und HTA}

Sowohl die Methode der EbM wie diejenige des HTA können genutzt werden, um die Entwicklung von Leitlinien zu unterstützen: EbM bei der Entwicklung klinischer Leitlinien (1), HTA bei Versorgungsleitlinien (2). Insofern sind Leitlinien eine Funktion von EbM und/oder HTA. Umgekehrt sind Leitlinien aber auch Instrumente der Implementation der beider Ansätze.

\section{Leitlinien und EbM}

EbM ist eine empirische Methode der Verwissenschaftlichung der Medizin durch eine Qualitätsrangfestlegung für medizinische Evidenzen. ${ }^{27}$ EbM ist gleichzeitig ein ärztliches Verhaltensprogramm: »Suche für alle deine Entscheidungen nach der besten verfügbaren Evidenz und stimme sie mit deiner klinischen Erfahrung ab. ${ }^{28}$ Die Praxis

27 Antes, Evidence-Based Medicine, Internist 1998, 39: 899-908; Sackett/Richardson/Rosenberg/Haynes, Evidenzbasierte Medizin, 1999; Raspe, Evidence-based medicine: Modischer Unsinn, alter Wein in neuen Schläuchen oder aktuelle Notwendigkeit?, ZaeFQ (1996) 90: 553-562; zu den rechtlichen Problemen Hart, Evidenz-basierte Medizin (EBM) und Gesundheitsrecht - Überlegungen zu rechtlichen Konsequenzen der Verwissenschaftlichung der Medizin, MedR 2000, $1 \mathrm{ff}$.

28 Siehe dazu Raspe, Von der Evidenz zur Empfehlung - zum Verhältnis von wissenschaftlicher Erkenntnis und Erfahrung, in: Hart (Hrsg.), Klinische Leitlinien und Recht, 2005, S. $63 \mathrm{ff}$, bes. $65-67,68,70$. 
der EbM bedeutet insofern die Integration individueller klinischer Expertise mit der externen Evidenz aus systematischer Forschung.

\section{a) EbM als Erkenntnisbasis}

EbM unterscheidet verschiedene Arten der Evidenz in der Medizin - angefangen bei Meta-Analysen von randomisierten kontrollierten Studien über einzelne randomisierte kontrollierte Studien, gut geplante nicht randomisierte Studien, Expertenwissen aufgrund kasuistischer Beobachtungen (Expertengruppen) bis hin zu klinischer Erfahrung (»Fachautoritäten «) - und statuiert einen Vorrang wissenschaftlicher Evidenz - geordnet nach dieser Rangskala - vor ärztlicher Erfahrung oder Intuition oder in anderer Formulierung von »probabilistischer vor hermeneutischer Evidenz« (F. Gerlach ${ }^{29}$ ) nach einer vergleichenden Qualitätsbeurteilung. EbM sucht also nach der »best available evidence «, ohne allerdings die anderen Arten der Evidenz gering zu schätzen oder gar auszuschließen. EbM macht insofern keine »Richtigkeitsvorgaben« für Behandlungsziele, sondern »Verfahrensvorgaben« für die Evaluation von medizinischen Erkenntnissen.

EbM präzisiert also methodisch die Anforderungen im Bereich der Erkenntnissammlung und Erkenntnisbewertung und das Verhältnis von wissenschaftlicher Erkenntnis und praktischer Erfahrung. Die Methode trägt durch Transparenzsteigerung erheblich zu einer Rationalisierung des Prozesses der Normgenerierung bei.

EbM schafft die Erkenntnisbasis für klinische Leitlinien, ohne damit den Gesamtprozess der Leitlinienerstellung als Normbildungsprozess vollständig zu determinieren. Bezogen auf den Standardbegriff füllt EbM als Erkenntnisbasis die Elemente »wissenschaftliche Erkenntnis und ärztliche Erfahrung« aus.

\section{b) Erkenntnisbasis und Konsensfindung}

Die Entwicklung der Maxime für die ärztliche Krankheitsbehandlung setzt als zweites Element ein akzeptiertes und gesichertes Verfahren der Verbindlichkeitsverabredung, der formalen Konsensfindung voraus. Solche formalen Konsensusverfahren bestehen typischerweise in einem formalen Gruppenprozess, der Delphimethode oder einer Konsensuskonferenz oder in einer Kombination dieser Verfahren. ${ }^{30}$

Qualitativ hochwertige klinische Leitlinien (S3; »evidenz-basierte Konsensus-Leitlinie «) werden nach Erkenntnisbasierung (EbM) aufgrund eines solchen Konsensfindungsprozesses entwickelt. Das Verfahren schließt den Prozess der Normbildung ab. Bezogen auf den Standardbegriff formalisiert das Konsensusverfahren das Element »professionelle Akzeptanz «. ${ }^{31}$

29 Qualitätsförderung in Praxis und Klinik - Eine Chance für die Medizin, 2001, S. 83 ff.

30 Black/Murphy/Lamping/McKee/Sanderson/Askham et al., Consensus development methods: a review of best practice in creating clinical guidelines, J Health Serv Res Policy 4[4], 236248. 1999; siehe auch Kopp/Encke/Hartig/Müller/Lorenz, Zur Empirie hochwertiger Leitlinien, in: Hart (Hrsg.), Klinische Leitlinien, S. 41 ff., bes. 49.

31 Vgl. Hart, Qualitätssicherung durch Leitlinien, VSSR 4/2002, 265 ff., bes. 274 f. 
Die Methode der EbM und das Verfahren der formalisierten Konsensusfindung gewährleisten zusammen wie kein anderes Verfahren, dass die Voraussetzungen der Standardentwicklung erfüllt werden. Hochwertige Leitlinien sind das seriöse Normbildungsverfahren in der Medizin. Diese Leitlinien setzen den Standard fest. Zum Normbildungsverfahren gehört selbstverständlich die angemessene Evaluierung und Aktualisierung der Leitlinie.

\section{Leitlinien und HTA}

Klinische Leitlinien sind die Voraussetzung für Versorgungsleitlinien und Versorgungsleitlinien sind die Instrumente der Umsetzung von HTA bzw. könnten sie sein. ${ }^{32}$ Die gegenwärtige Praxis ist hinter der Klarheit dieser Funktionsaussage allerdings erheblich zurück.

Klinische Leitlinien stellen einen Ausschnitt aus dem Bewertungskonglomerat der Versorgungsleitlinien dar. Jene betreffen allein die medizinische Empfehlung der Behandlung einer bestimmten Erkrankung. Insofern ist EbM ebenfalls ein Ausschnitt aus dem - mehr oder weniger umfassenden - Technologiebewertungsanspruch von HTA. ${ }^{33}$ HTA baut auf EbM auf, trifft aber darüber hinausgehende Bewertungen insbesondere unter dem Aspekt Versorgungs- im Verhältnis zu Individualnutzen und vor allem Effizienz, die für die klinischen Leitlinien prinzipiell keine Rolle spielt.

Hinsichtlich der klinischen Leitlinien war unsere Aussage: Die hochwertige Leitlinie wird zum Zentrum der Normbildung in der Medizin. Für die Versorgungsleitlinien kann eine solche Aussage nur sehr beschränkt getroffen werden. Die Versorgungsleitlinie kann hinsichtlich ihrer klinischen Elemente dieselbe Qualität haben, hinsichtlich der darüber hinausgehenden Elemente gilt dies nur begrenzt, weil dort sowohl für die Erkenntnisbasis wie für das Konsensusverfahren Defizite existieren bzw. andere Bedingungen gelten. Versorgungsleitlinien sind Regulierungsinstrumente in der Gesundheitsversorgung und deshalb als Normen nur im Rahmen einer bestehenden Gesundheitsrechtsverfassung gültig.

a) HTA als Erkenntnisbasis

EbM stellt einen Teil der Erkenntnisbasis, die HTA benötigt, wenn es eine umfassende Bewertung einer Gesundheitstechnologie, die in einem Versorgungssystem genutzt wird, abgeben soll. EbM gewährleistet die medizinische Basis der Beurteilung, also im Wesentlichen die für die Nutzenbewertung. ${ }^{34}$ Darüber hinaus bedarf es der Erkenntnisse über die Versorgungseffektivität und die Effizienz (Kosten-Wirksamkeit) der Technologie für das Versorgungssystem und der Informationen über soziale, ethische und rechtliche Implikationen für den Fall der »Zulassung « der Technologie.

32 Hart, Qualitätssicherung durch Leitlinien, VSSR 4/2002, 265 ff., bes. 275, 278.

33 Dazu Hart, Der regulatorische Rahmen der Nutzenbewertung: Vom Arzneimittelrecht zum HTA-Recht, MedR 2004, 469 ff., bes. $472 \mathrm{f}$.

34 Dazu das Schema bei Hart, Der regulatorische Rahmen der Nutzenbewertung: Vom Arzneimittelrecht zum HTA-Recht, MedR 2004, 469 ff., bes. 473. 
Ob die Qualitätsrangskala von EbM, die für die Medizin entwickelt wurde, auf die weiteren genannten Informationen aus den verschiedenen Disziplinen übertragen werden kann, sei dahingestellt, ist aber zu bezweifeln - mindestens erscheinen Anpassungen der Qualitätskriterien erforderlich. ${ }^{35}$ Eine EbM-vergleichbare akzeptierte Methode der Informationsgenerierung steht jedenfalls nicht selbstverständlich zur Verfügung.

\section{b) HTA: Erkenntnisbasis und Konsensfindung}

Problematischer noch erscheint allerdings die fehlende Übereinkunft über den Prozess der Konsensusherbeiführung in der HTA-Wissenschaft und -Praxis. Die formalen Konsensusverfahren der Leitlinien-Medizin sind bisher auf den interdisziplinären und multiprofessionellen Konsensfindungsprozess in den Gesundheitswissenschaften bzw. in den bewertenden Versorgungsinstitutionen des Gesundheitssystems nicht übertragen worden oder dort jedenfalls nicht etabliert. Der Schritt vom (informationssammelnden und empfehlenden) HTA-Report zur institutionellen HTA-Entscheidung ist hinsichtlich des Konsensusprozesses auch nicht ansatzweise institutionalisiert. Ob man bei der Erstellung von HTA-Reports hinsichtlich der nicht-medizinischen Bewertungen ebenfalls Konsensusverfahren zu berücksichtigen hätte, scheint mir überlegenswert.

Hinsichtlich der institutionellen HTA-Entscheidung (also innerhalb des Versorgungssystems) sind regulatorische Vorgaben gemacht (Abstimmungsprozeduren im Gemeinsamem Bundesausschuss (G-BA) gem. § 91 SGB V), die anderen Gesetzen folgen als den bekannten Konsensusverfahren; es handelt sich um rechtsanwendungspolitische Entscheidungen aufgrund von Abstimmungen in der gemeinsamen Selbstverwaltung unter Mitberatung von Patienten. Es geht insofern um normkonkretisierende Entscheidungen insbesondere des G-BA, die nach der ständigen Rechtsprechung des BSG nur einer begrenzten sozialgerichtlichen Kontrolle unterliegen (untergesetzliche Normsetzung). ${ }^{36}$

Aus der englischen HTA-Literatur ist die Unterscheidung zwischen assessment und appraisal bekannt ${ }^{37}$, die dort die institutionelle Unterscheidung der Aufgaben von insbesondere universitären (bewertenden) HTA-Gruppen auf der einen und (empfehlenden) NICE sowie der (entscheidenden) politischen Institutionen auf der anderen Seite beschreibt. In der deutschen Diskussion um das Institut für Qualität und Wirtschaftlichkeit im Gesundheitswesen (IQWiG) hat man diese Unterscheidung teilweise auf

35 Vgl. dazu Busse/Orvain/Velasco/Perleth/Drummond/Gürtner/J ørgensen/Jovell/Malone/ Rüther/Wild, Best Practice in Undertaking and Reporting Health Technology Assessments, Int. J. of Technology Assessment in Health Care 18:2, 2002, 361-422, bes. $384 \mathrm{ff}$.

36 Engelmann, Untergesetzliche Normsetzung im Recht der gesetzlichen Krankenversicherung durch Verträge und Richtlinien, NZS 2000, 1 ff., 76 ff.; ders., Das Rechtskonkretisierungskonzept des SGB V und seine dogmatische Einordnung durch das Bundessozialgericht, in Hart (Hrsg.), Ärztliche Leitlinien: Empirie und Recht professioneller Normsetzung, 2000, S. 199 ff.; Mengel, Sozialrechtliche Rezeption ärztlicher Leitlinien, 2004, S. $204 \mathrm{ff}$.

37 Stevens/Milne, Health technology assessment in England and Wales, International Journal of Technology Assessment in Health Care 2004; 20: 11-24; Taylor, National Institute for Clinical Excellence (NICE). HTA rhyme and reason?, Int J Technol Assess Health Care 2002; 18: 166-170. 
das Verhältnis von IQWiG und G-BA übertragen. ${ }^{38}$ Richtig daran ist, dass das IQWiG empfiehlt und der G-BA entscheidet; das IQWiG kann selbst HTA-Berichte erstellen, kann sie aber auch in Auftrag geben, so dass sich dann eine »englisch-walisische Struktur « ergibt. Regulatorisch betrachtet können (externe oder interne) HTA-Berichte zur Grundlage von IQWiG-Empfehlungen und G-BA-Entscheidungen werden, die die Form von Richtlinien als Versorgungsleitlinien annehmen. Existierende hochwertige klinische Leitlinien gehen in sie als »Standard« ein.

\section{Leitlinie und Standard im Medizinrecht}

Das Medizinrecht als Teil des Gesundheitsrechts integriert alle rechtlichen Normen, die den Gegenstand Medizin betreffen. Einer seiner wichtigsten Bereiche ist das dienstleistungsbezogene Vertrags- und Deliktsrecht (Heilberufs[haftungs]recht). ${ }^{39}$

In diesem zivilen ärztlichen Berufsrecht identifizieren wir die ärztlichen Pflichtengefüge trotz unterschiedlicher Entstehungsgründe in der Regel als Behandlung, Information/Aufklärung/Beratung, Dokumentation und Organisation. Die Einsichtsgewährung in die Krankendokumentation ist Patientenrecht und ärztliche bzw. Trägerpflicht zugleich. Der Sache nach behandeln wir die unterschiedlichen Entstehungsgründe Vertrag und Delikt, ausgeübte Vertragsinhaltsfreiheit und berufliche Verkehrspflicht, gleich: Der Inhalt der vertraglichen ärztlichen Hauptleistungspflicht Behandlung entspricht dem der deliktischen ärztlichen Verkehrspflicht Behandlung. Beide werden und das ist die Konsequenz der bisherigen Ausführungen zu den klinischen Leitlinien - durch den Inhalt der hochwertigen Leitlinie festgesetzt.

\section{Pflichteninhalt: Behandlung}

Im Gegensatz zur überwiegenden Meinung in Literatur und im Gegensatz zur Rechtsprechung $^{40}$ wird von einer Mindermeinung in der Literatur der Pflichteninhalt der

38 Hart, Der regulatorische Rahmen der Nutzenbewertung: Vom Arzneimittelrecht zum HTARecht, MedR 2004, 469 ff., 476.

39 Hart/Francke, Einführung, Gesundheitsrecht, 5. Aufl. 2003, Beck-Texte im dtv, S. IX ff., bes. X f., XIII.

40 Statt vieler Katzenmeier, Arzthaftung, 2002, S. 180 ff.; Deutsch/Spickhoff, Medizinrecht, 5. Aufl. 2003, 5. Aufl. 2003, Rdnr. 125 ff.; Giesen, Arzthaftungsrecht, 4. Aufl. 1995, Rdnr. 99 ff., bes. 107 ff.; Walter, Spezialisierung und Sorgfaltsstandard im Arzt- und Anwaltshaftungsrecht, 2004, S. 152 ff.; ebenso die st. Rspr. z. B. BGH NJW 2001, $1786=$ VersR 2001, 646 - Fahrlässigkeit und Standard und Steffen/Dressler, Arzthaftungsrecht, 8. Aufl. 1999, Rdnr. 151: »Das Recht muß ihn zwar kontrollieren und gegebenenfalls korrigieren.« Die Entscheidung BGH NJW 2003, 2311, 2313 - Unterlassen einer Hysteroskopie als ärztlicher Behandlungsfehler bezieht sich zwar auf die vorangehend zitierte, könnte aber durchaus auch in die Richtung einer Identität von medizinischem und rechtlichem Standard weisen. Jüngst auch MünchKomm-Wagner $§ 823$ Rdnr. 678 mit der kämpferischen Formulierung »Die Schwierigkeiten, das allgemeine Sorgfaltsgebot auf den Einzelfall anzuwenden, rechtfertigen es allerdings nicht, die Definitionsmacht über den Umfang ärztlicher Sorgfaltspflichten an die medizinische Profession abzutreten und sie dem ärztlichen Berufsethos oder der faktischen Übung im Bereich der Heilbehandlung zu überantworten.« mit überraschendem Zitat Hart - da Sorgfalt, Berufsethos und Übung völlig unterschiedliche 
vertraglichen Hauptleistungs- und der deliktischen Verkehrspflicht Behandlung allein medizinisch bestimmt. ${ }^{41}$ Die Mehrheitsmeinung lässt sich zwar eine rechtliche Korrektur des medizinischen Standards offen, nimmt diese Möglichkeit allerdings nur im Rahmen der Organisationspflichten wahr, die richtigerweise aus dem Behandlungsbegriff auszugliedern sind.

Auch die jüngeren Stellungnahmen von Katzenmeier ${ }^{42}$ oder von Wagner ${ }^{43}$ nehmen m. E. in unterschiedlicher Weise, aber dennoch beide die modernen Entwicklungen der Medizin, die mit dem Stichwort EbM und Leitlinien verbunden und hier geschildert sind, nicht hinreichend zur Kenntnis. ${ }^{44}$ Der Standardbegriff der Medizin ist ebenso eine Norm guter Behandlung, wie die vertragliche Hauptleistungspflicht des Arzt/Patient-Vertrages die Norm guter Behandlung zugrunde legt. Der Patient erwartet eine Behandlung nach dem anerkannten Stand der medizinischen Wissenschaft und Praxis und insofern bestimmt der medizinische Standard, auf den der Arzt verpflichtet ist, den Inhalt der Hauptleistung. Und diesen Standard setzt die hochwertige klinische Leitlinie fest. Eine S3-Leitlinie ist der Standard, ist der Vertragsinhalt und der Inhalt der Verkehrspflicht. ${ }^{45}$ Die Seriosität des Verfahrens der Erstellung der »evidenzbasierten Konsensus-Leitlinie« gewährleistet eine höhere Qualität der »Standardfeststellung « als jedes andere Verfahren sonst. Kein Sachverständiger dürfte eine höhere Qualität gewährleisten können und es geht gerade nicht um das Abtreten einer Definitionsmacht, ihre Überantwortung an die ärztliche Ethik oder gar an die faktische Übung. ${ }^{46}$ Die durch die Profession konsensual bewertete best available evidence ist auf Verfahrensqualität rechtlich kontrollierbar (»Verfahrenskontrolle«), aber rechtlich nicht inhaltlich korrigierbar - es fehlt dem Recht jegliche inhaltliche Korrekturkompetenz.

Dinge sind und es bei den Leitlinien um Normen guter Behandlung (Standards) geht, ist die Konsistenz der Aussage mindestens zweifelhaft .

41 Überblick über den Meinungsstand bei Hart, Diagnosefehler. Seine Verortung als Behandlungsfehler und die Verpflichtung zur Aufklärung, in: Brüggemeier (Hrsg.), Liber Amicorum Eike Schmidt, 2005, S. 69-97; ders., Arzneimittelinformation zwischen Sicherheitsund Arzthaftungsrecht. Fach- und Gebrauchsinformation, ärztliche Aufklärung und Pflichtverletzung, MedR 2003, 603 ff., bes. 607 ff.

42 Arzthaftung, 2002, S. $180 \mathrm{ff}$.

43 MünchKomm § 823 Rdnr. 678.

44 Auch die jüngere spezifische Leitlinienliteratur zieht die hier vorgetragene Konsequenz noch nicht; vgl. Igloffstein, Regelwerke für die humanmedizinische Individualbehandlung, 2003; Nowak, Leitlinien in der Medizin, 2002; Arbeitsgemeinschaft Rechtsanwälte im Medizinrecht e.V. (Hrsg.). Leitlinien, Richtlinien und Gesetz - Wieviel Reglementierung verträgt das Arzt-Patienten-Verhältnis?, 2003 und dort insbesondere die Aufsätze von Stegers, Richtlinien des Bundesausschusses Ärzte Krankenkassen - Standard und Haftung, S. 11 ff. und Bergmann, Leitlinien und Haftung, S. 65 ff.

45 In diese Richtung auch Diederichsen, Zur Bedeutung ärztlicher Leitlinien für die Haftung aus einem Behandlungsverhältnis, in: Hart (Hrsg.), Klinische Leitlinien und Recht, S. 105 ff., bes. 108 .

46 MünchKomm-Wagner § 823 Rdnr. 678. 


\section{Pflichtverletzung: Behandlungsfehler}

Die Abweichung von der hochwertigen Leitlinie ist deshalb in der Konsequenz der Behandlungsfehler - eine Vermutung, die auf zwei Wegen widerlegbar ist: durch die Begründung der Erforderlichkeit einer individuellen Abweichung oder die Begründung der »Überholung « der Leitlinie aufgrund neuer Erkenntnis. ${ }^{47}$ Letzteres ist die Folge der Erforderlichkeit der Evaluation und Aktualisierung der hochwertigen Leitlinie. Die Leitlinie ist die rechtlich rezipierte medizinische Norm.

Diese Aussage gilt selbstverständlich nur bei solchen Leitlinien, die auch tatsächlich einen klar handlungsempfehlenden und nicht nur einen informierenden Charakter haben, möglicherweise nur Behandlungsalternativen aufzeigen oder nach der Stärke des Empfehlungsgrades differenzieren (von stark nach schwach), also einen schwachen Empfehlungsgrad aussprechen.

Die Anwendung der Leitlinie (Norm) auf den Einzelfall ist Subsumtion. Im ersten Schritt ist daher festzustellen, ob die Leitlinien-Indikation und die Patientenerkrankung übereinstimmen, ob und welche Behandlungsempfehlung abgegeben wird und ob Behandlungsempfehlung und ärztliches Handeln sich entsprechen. Sind diese Voraussetzungen erfüllt, kommt ein Behandlungsfehler nicht in Betracht. Ist die Indikation nicht gegeben, kommt eine Behandlung nach der Leitlinie nicht in Betracht; erfolgt sie dennoch, kann die Leitlinie für die Frage des Behandlungsfehlers nicht herangezogen werden. Es ist nach einer einschlägigen Leitlinie bzw. nach dem entsprechenden Standard zu suchen, falls keine Leitlinie einschlägig ist.

Weicht die Behandlung trotz Indikation von der Leitlinie $a b$, kann der Behandlungsfehlertatbestand nur dann vermieden werden, wenn entweder neue Erkenntnisse vorliegen oder/und (deshalb) neue Bewertungen ${ }^{48}$ vorgenommen werden (müssen) oder wenn individuelle patientenbezogene Umstände die empfohlene Behandlung nicht erlauben. Der Notfall erfordert, wenn die Leitlinie dafür keine Empfehlung enthält, einen anderen Standard.

Die Ausführungen verdeutlichen, dass die Leitlinie den Sachverständigen im Haftpflichtprozess nicht überflüssig macht, aber sie konzentriert seine Aufgabe ${ }^{49}$ Innerhalb der Indikation ist sie seine »Richtschnur«; er muss sie seinem Auftrag zugrunde legen und anwenden.

\section{Zum Stand der Rechtsprechung}

Die Rechtsprechung der letzten Jahre ist uneinheitlich, gibt den Leitlinien teilweise keine Bedeutung, differenziert teilweise Richtlinien unterschiedlicher Art und Leitlinien nicht voneinander und nimmt insgesamt von der medizinischen Empirie der

47 Noch nicht in dieser Konsequenz Hart, Leitlinien und Haftungsrecht - Inkorporation, Rezeption und Wissensbasis in Wissenschaft und Praxis, in: Hart (Hrsg.), Klinische Leitlinien, S. $81 \mathrm{ff} ., 96 \mathrm{ff}$.

48 Der alten oder der neuen Evidenz.

49 Dressler, Ärztliche Leitlinien und Arzthaftung, in: Brandner/Hagen/Stürner (Hrsg.), FS für Karlmann Geiß zum 65. Geburtstag, 2000, S. 379, 384; Weidinger, Leitlinien und ärztliche Berufshaftpflicht, Der Internist 6·2001, M118-123, bes. 121. 
Leitlinien kaum Kenntnis. Der BGH hat sich bisher mit Leitlinien nicht zu befassen gehabt. ${ }^{50}$ Die Rechtsprechung im Übrigen ist »standardverhaftet «.

Eine Entscheidung des OLG Naumburg ${ }^{51}$ ist im Hinblick auf die arzthaftungsrechtliche Bedeutung von ärztlichen Leitlinien signifikant. Es handelt sich wohl um das erste obergerichtliche Urteil zu ärztlichen Leitlinien. Andere Urteile haben sich mit Richtlinien oder Empfehlungen beschäftigt. ${ }^{52}$ Die Aussage des OLG Naumburg, dass die bisher existierenden Leitlinien der AWMF »derzeit lediglich Informationscharakter für die Ärzte selbst haben und haben sollten«, ist sicherlich zu diesem Zeitpunkt wegen der geringen Zahl der nach dem neuen Verfahren zustande gekommenen hochwertigen Leitlinien grundsätzlich berechtigt. Dasselbe gilt für die Aussage, Leitlinien seien »wegen ihres abstrakten Regelungsgehalts« nicht geeignet, ein Sachverständigengutachten zu ersetzen. Das verweist auf die Notwendigkeit der Anwendung der Leitlinie im individuellen Behandlungsfall.

Eine jüngste Entscheidung des $\mathrm{KG}^{53}$ beschäftigt sich mit den Mutterschaftsrichtlinien des Bundesausschusses der Ärzte und Krankenkassen - also in der hiesigen Terminologie eine Versorgungsleitlinie in der Form einer sozialrechtlichen Richtlinie und misst diesen als Regelwerk eine Bedeutung bei, die sie nach den vorangehenden Ausführungen so jedenfalls nicht haben können, nämlich die Funktion der haftungsrechtlichen Standardfestlegung. ${ }^{54}$ Diese Funktion könnten sie nur dann haben, wenn sie in ihrer empfehlenden medizinischen Aussage auf einer hochwertigen Leitlinie basierten. Die weitere Äußerung, dass der sozialrechtliche (GKV-)Standard und der haftungsrechtliche Standard übereinstimmten, die Maßstäbe der Gesetzlichen Krankenversicherung auch diejenigen der Privaten Krankenversicherung bestimmten, ist jedenfalls unterkomplex. ${ }^{55}$

Die veröffentlichten Gerichtsentscheidungen ${ }^{56}$ zeigen, dass die Leitlinien bisher in der Rechtspraxis kaum angekommen sind. ${ }^{57}$ Die Kenntnis über die Leitliniendebatte scheint gering und die systematische Auseinandersetzung anhand eines Einzelfalles ist bisher nicht erfolgt. Der BGH hat noch keinen »passenden« Fall gehabt und die

50 Siehe insgesamt zur Rechtsprechung Diederichsen, Zur Bedeutung ärztlicher Leitlinien, S. 105 ff., bes. $107 \mathrm{ff}$.

51 OLG Naumburg MedR 2002, 471 (bearbeitet von Hart).

52 OLG Düsseldorf VersR 2000, 1019 - Leitlinien einer Fachgesellschaft zu Hygieneproblemen; OLG Hamm VersR 2000, 1373 - Leitlinien für Wiederbelebung und Notfallversorgung; OLG Hamm NJW 2000, 1801 - Richtlinien der BÄK.

53 KG NJW 2004, 691 - Mutterschaftsrichtlinien.

54 Ähnlich Spickhoff, Die Entwicklung des Arztrechts 2003/2004, NJW 2004, 1710 ff., 1714.

55 Dazu Hart, Qualitätssicherung durch Leitlinien, VSSR 2002, 265, 290 ff.

56 Siehe auch OLG Düsseldorf VersR 2000, 1019 - Leitlinien einer Fachgesellschaft zu Hygieneproblemen; OLG Hamm VersR 2000, 1373 - Leitlinien für Wiederbelebung und Notfallversorgung; OLG Hamm NJW 2000, 1801 - Richtlinien der BÄK; OLG Stuttgart MedR 2002, 650 ff., 652 f. - Leitlinien der Deutschen Gesellschaft für Chirurgie (bearbeitet von Kern).

57 Siehe demnächst Hart (Hrsg.), Ärztliche Leitlinien im Medizin- und Gesundheitsrecht Recht und Empirie professioneller Normbildung, (erscheint Mitte 2005; es handelt sich um die Veröffentlichung der Ergebnisse eines Forschungsprojektes, aus dem zuvor der Tagungsband Hart (Hrsg.), Klinische Leitlinien und Recht, 2005, hervorgegangen ist) . 
sachverständige Vorbereitung der Gerichte auf die Fallentscheidung bei Leitlinienrelevanz scheint ebenfalls unzureichend.

\section{Weitere Konsequenzen}

Leitlinien dieser Qualität werden orientierende Wirkungen insbesondere für die Sicherungs- oder therapeutische Aufklärung und für die Patientenberatung entfalten. Sie können für die Verlaufs- und Risikoaufklärung als Hilfe herangezogen werden. Beides wird insbesondere dann der Fall sein können, wenn es Patientenversionen der Leitlinien gibt, was z. B. für die Leitlinien der DEGAM ${ }^{58}$ gilt.

Die Leitlinie kann die Dokumentation erleichtern, stellt aber auch Anforderungen: Die Abweichungsgründe sind zu dokumentieren ${ }^{59}$, will der Arzt die Fehlerindizwirkung bei Abweichung von der standardfestsetzenden Leitlinie vermeiden. ${ }^{60}$ Der Grund für diese Pflicht zur Dokumentation der Abweichungsbegründung liegt im Verlassen des »standardgemäßen« Behandlungsweges, den die Leitlinie festgelegt hat. Fehlt es an der Dokumentation der Abweichungsbegründung und kann der Arzt diese Gründe mit anderen Beweismitteln nicht nachweisen, wird der Behandlungsfehler durch die Abweichung »fingiert«. Dies folgt aus der Übertragung der ständigen Rechtsprechung zur Verletzung von Dokumentationspflichten. ${ }^{61}$ Mangelnde Dokumentation führt wegen der Beeinträchtigung von Nachweismöglichkeiten des Patienten zur den Arzt belastenden Vermutung, die nicht dokumentierte Handlung sei auch nicht erfolgt. ${ }^{62}$ Die Handlung besteht in der Begründung für die Abweichung, deren Möglichkeit dann negiert werden muss.

Der Organisationspflichtige hat sicherzustellen, dass innerhalb seiner Institution die Voraussetzungen der leitliniengemäßen Behandlung zu gewährleisten sind. Kann er das nicht, muss er eine nicht-leitliniengemäße - also eine nicht-standardgemäße - Behandlung verhindern (organisatorische Prävention). ${ }^{63}$

Im Arzthaftpflichtprozess hat das Gericht die anzuwendende Norm festzustellen und zu subsumieren. In der Konsequenz der bisherigen Ausführungen bedeutet dies:

$>$ Ob eine (Vertrags- oder Verkehrs-)Pflicht und mit welchem Inhalt besteht, ist durch das Gericht festzustellen. ${ }^{64}$ Die ärztliche Pflicht zur standardgemäßen Behandlung

58 Deutsche Gesellschaft für Allgemeinmedizin und Familienmedizin.

59 Dressler, Die Bedeutung von ärztlichen Leitlinien im Rechtsstreit, in: Hart (Hrsg .), Ärztliche Leitlinien, S. 161 ff., bes. 167 ff.; Hart MedR 1998, 8, 14; ders., Leitlinien und Haftungsrecht, in: Hart (Hrsg.), Ärztliche Leitlinien, S. 137 ff.

60 Hart, Leitlinien und Haftungsrecht, in: Hart (Hrsg.), Ärztliche Leitlinien, S. 137 ff., 145; Steffen/Dressler, Arzthaftungsrecht, Rdnr. 161b.

61 Steffen/Dressler, Arzthaftungsrecht, Rdnr. 498 ff., $455 \mathrm{ff}$.

62 BGHZ 72, 132, 138 - Blinddarmoperation; 88, 230 - Anfängeroperation.

63 Vgl. BGH NJW 1991, 1543 f. - Beschaffung eines Medikaments vor Operation; zu Organisationspflichten dieser Art Hart, »Organisationsaufklärung « - Zum Verhältnis von Standardbehandlung, Organisationspflichten und ärztlicher Aufklärung, MedR 1999, 47-50.

64 Siehe E. Schmidt AK-ZPO, Einl. Rdnr. 75 ff.; ders., Richteramt und Parteilasten bei der Verbandsklage nach dem deutschen AGB-Gesetz, Festschrift für Max Keller zum 65. Geburtstag, hrsg. v. Forstmoser/Giger/Heini/Schluep, 1989, S. 661, 669 ff. hat dafür den Begriff der Normtatsachen geprägt. 
(= leitliniengemäßen Behandlung) ist mit Hilfe des Sachverständigen durch das Gericht zu klären. ${ }^{65}$ Es ist nicht die Aufgabe einer Partei, das Risiko der Rechtsnormermittlung zu tragen. Es ist Aufgabe der beanspruchenden Partei, den Nachweis der tatsächlichen Verletzung dieser Pflicht zu führen.

$>$ Das Gericht hat deshalb zu klären, ob für diese Erkrankung eine hochwertige Behandlungsleitlinie existiert und ob sie eine Behandlungsmaxime aufstellt.

$>$ Der Sachverständige ist deshalb in jedem Falle verpflichtet, das Bestehen hochwertiger Leitlinien zu prüfen ${ }^{66}$ und sie im Vorhandensfalle seinem Gutachten zugrunde zu legen. ${ }^{67}$

$>$ Weicht die Behandlung von der Leitlinienempfehlung ab (zum Nachweis des Patienten), steht der Behandlungsfehler bis zur evtl. Entlastung durch den Arzt (zu dessen Nachweis: Nicht-Anwendbarkeit; Erforderlichkeit der Abweichung) fest. ${ }^{68}$

Die Anforderungen an die Substanziierungslast durch den geschädigten Patienten im Arzthaftpflichtprozess für den Behandlungsfehler sind maßvoll, ${ }^{69}$

»weil vom Patienten regelmäßig keine genaue Kenntnis der medizinischen Vorgänge erwartet und gefordert werden kann. Die Partei darf sich auf Vortrag beschränken, der die Vermutung eines fehlerhaften Verhaltens des Arztes aufgrund der Folgen für den Patienten gestattet ..."

Die Behauptung eines Verstoßes gegen eine ärztliche Leitlinie erfüllt diese Anforderungen, wenn die Leitlinie die in Rede stehende Erkrankung betrifft.

Jenseits des Behandlungsfehlers könnten sich aus dem hier vertretenen Ansatz auch beweisrechtliche Konsequenzen hinsichtlich des Nachweises der haftungsbegründenden Kausalität ergeben. Die Abweichung von der hochwertigen Leitlinie als groben Behandlungsfehler zu bezeichnen, was im Anknüpfung an die jüngste Rechtsprechung des $\mathrm{BGH}^{70}$ jedenfalls nicht unmöglich erscheint, ${ }^{71}$ wäre dennoch eine zweifelhafte

65 Ausführlich Velten, Der medizinische Standard im Arzthaftungsprozess - Ein Beitrag zu Umfang und Grenzen der Darlegungslast von Arzthaftungsklägern bezüglich der Standards medizinischer Heilbehandlung, 2000, S. 98 ff., 107 ff.

66 Hansis in: Stegers/Hansis/Alberts/Scheuch, Der Sachverständigenbeweis im Arzthaftungsrecht, 2002, Rdnr. 335 ff.; Stegers in: Stegers/Hansis/Alberts/Scheuch, Der Sachverständigenbeweis, Rdnr. 144 ff., bes. 159 ff.

67 Ebenso Dressler, Ärztliche Leitlinien und Arzthaftung, S. 379, 384; Weidinger, Leitlinien und ärztliche Berufshaftpflicht, Der Internist 6·2001, M118-123, bes. 121.

68 Eine Indizwirkung nehmen an: Deutsch/Spickhoff, Medizinrecht, Rdnr. 163; Dressler, Ärztliche Leitlinien und Arzthaftung, S. 379, 382 f.; Diederichsen, Zur Bedeutung ärztlicher Leitlinien, S. 105 ff., 108.

69 Jüngst auch für die Rechtslage nach der Schuldrechtsmodernisierung wiederholend BGH VersR 2004, 1177 = MedR 2005, 37 - Trümmerbruch mit umfangreichen Angaben zur Rechtsprechung.

70 BGH VersR 2004, 909 = NJW 2004, 2011 = JZ 2004, 1029 - Beckenringfraktur; dazu allgemein Katzenmeier, Arzthaftung, S. 439 ff.; zur Entscheidung Katzenmeier, Anm. JZ 2004, 1030 ff.; Spickhoff, Grober Behandlungsfehler und Beweislastumkehr, NJW 2004, 2345 ff.; jüngst BGH VersR 2005, 228 - grobe Verletzung der Pflicht zur Sicherungsaufklärung führt regelmäßig zur Umkehr der objektiven Beweislast für die haftungsbegründende Kausalität . 
Verknüpfung von Fehler und Verletzung/Schaden, weil der wahre Grund für eine solche Verknüpfung in der durch die Abweichung von der Leitlinie induzierten Risikoerhöhung für den Patienten liegt. ${ }^{72}$ Der Sache nach ist dieser Gedanke auch in der jüngsten Entscheidung ${ }^{73}$ zur Beweislastumkehr bei Verletzung der Verpflichtung zur therapeutischen Aufklärung enthalten: Jede Unterlassung riskiert eine Risikoerhöhung, die durch ein der Sicherungsaufklärung entsprechendes Verhalten wahrscheinlich beherrschbar gewesen wäre. Es geht um die Sanktion für die Verhinderung der Chance einer patientenschützenden Risikoprävention. ${ }^{74}$

Alle dargestellten Konsequenzen gelten ausschließlich für die »evidenz-basierten Konsensus-Leitlinien «. Nur ihre Kriterien- und Verfahrensseriositäten begründen die Tragfähigkeit der »automatischen« rechtlichen Rezeption der medizinischen Bewertung.

\section{Leitlinie und Standard im Gesundheitsrecht}

Parallel zu den vorangehenden medizinrechtlichen Ausführungen lassen sich für das Gesundheitsrecht ähnliche Strukturaussagen begründen, auch wenn die Differenzen beider Rechtsgebiete nicht nivelliert werden dürfen. ${ }^{75}$ Unterschiede gelten insbesondere hinsichtlich der Regelungs- und Entscheidungsebenen. ${ }^{76}$ Die Parallelen gelten für das Leistungsrecht der individuellen Behandlung (Einzelentscheidungen der Leistungserbringer), soweit es sich um deren Zweckmäßigkeit (= Qualität und Wirksamkeit = Stand der medizinischen Erkenntnisse unter Berücksichtigung des medizinischen Fortschritts; § 2 I 3 SGB V) handelt; Abweichungen sind im Qualitätssicherungsrecht bzw. bei den »Zulassungsnormen « des SGB V, wo es um allgemeine, den Leistungsrahmen festlegende Entscheidungen geht, geboten. Im Einzelnen ist in der spezialisierten Literatur vieles offen oder streitig. Im Zentrum der folgenden Ausführungen steht der Zusammenhang von Leitlinien, HTA und Gesundheitsrecht und damit gegenüber dem Medizinrecht die Ausweitung der Bewertungskriterien für medizinische Leistungen, die Unterschiedlichkeit der Regelungsebenen und die Besonderheiten der normsetzenden Institutionen.

71 Dagegen OLG Stuttgart MedR 2002, 650 ff, 652 f. - Leitlinien der Deutschen Gesellschaft für Chirurgie (bearbeitet von Kern); eine Zwischenposition findet sich auf den jeweiligen Fall und die Bekanntheit der Leitlinie bezogen bei Dressler, Ärztliche Leitlinien und Arzthaftung, S. 379, 386.

72 In diese Richtung (ohne den Bezug auf Leitlinien) auch Katzenmeier JZ 2004, 1032; ders., Arzthaftung, S. $464 \mathrm{ff}$.

73 BGH VersR 2005, 228, 229 - grobe Verletzung der Pflicht zur Sicherungsaufklärung führt regelmäßig zur Umkehr der objektiven Beweislast für die haftungsbegründende Kausalität .

74 Ein solches Sanktionselement enthalten trotz Bestreitens alle »grob«-Anknüpfungen von Beweislastumkehren.

75 Siehe insbesondere Francke, Leitlinien und Sozialrecht, in: Hart (Hrsg.), Klinische Leitlinien, 2005, S. 123 ff. sowie Clemens, Leitlinien und Sozialrecht - Rezeption von Leitlinien durch Rechtssetzung und Rechtsprechung, ebendort, S. $147 \mathrm{ff}$.

76 Francke, Leitlinien und Sozialrecht, S. 123 ff., bes. 126 ff.; insgesamt Mengel, Sozialrechtliche Rezeption, S. $185 \mathrm{ff}$. 


\section{Leitlinie bestimmt Zweckmäßigkeit}

Wenn die Ausgangsthese richtig ist, dass die hochwertige ärztliche Leitlinie den Standard festsetzt, dann muss auch im GKVR eine solche Leitlinie mit dem Standard der Behandlung im Einzelfall und damit der Zweckmäßigkeit der Behandlung identifiziert werden. ${ }^{77}$ Eine der evidenz-basierten Konsensus-Leitlinie entsprechende Behandlung entspricht § 2 I 3 SGB V und erfüllt die Zweckmäßigkeitsvoraussetzungen der §§ 12 I, 27 I SGB V im Rahmen des Wirtschaftlichkeitsgebotes, sofern nicht gesetzliche Leistungsausschlüsse oder die generellen und abstrakten Regelungen des Leistungserbringungsrechts und auf ihnen beruhende Entscheidungen entgegenstehen. ${ }^{78}$ Die Vorbehalte von Francke, der selbst eine Vermutungswirkung verneint, gegenüber dieser strikten Aussage ${ }^{79}$ beziehen sich nicht auf die Zweckmäßigkeit, sondern nur auf die weiteren Kriterien im Rahmen der Wirtschaftlichkeit.

Die Rede ist allein vom Typus der hochwertigen ärztlichen Leitlinie, nicht von Versorgungsleitlinien. Erstere kann eine zweckmäßigkeitsbestimmende Wirkung nur als medizinische entfalten oder wenn sie ohne Abstriche mit ihren medizinischen Inhalten in die Versorgungsleitlinie eingegangen wäre. Hinsichtlich aller sonstigen Bewertungen der Wirtschaftlichkeit von Behandlungen im GKVR existiert kein »Rezeptionszwang $~^{80}{ }^{80}$ Die ärztliche Leitlinie hat keine HTA-Qualität.

Der Vertragsarzt, der der evidenzbasierten Konsensus-Leitlinie folgt, ist hinsichtlich der Zweckmäßigkeit der Behandlung gebunden und gleichzeitig gedeckt, bezüglich der Wirtschaftlichkeit im engeren Sinne bedarf es weiterer Bewertungen. ${ }^{81}$ Einschränkungen dieser Aussage können sich aufgrund des Leistungserbringungsrechts ergeben (z. B. fehlende Zulassung oder Ausschluss einer Untersuchungs- und Behandlungsmethode gem. § 135 I SGB V).

Bezüglich des Beurteilungsspielraums bzw. Normsetzungsermessens des $G-B A$ beispielsweise bei Entscheidungen nach $\S \S 135$ I, 137 c SGB V gilt m. E. dasselbe. Der G-BA ist hinsichtlich des medizinischen Teils der hochwertigen Leitlinie (= Zweckmäßigkeit) einerseits gebunden und andererseits hinsichtlich der Wirtschaftlichkeit im engeren Sinne ungebunden und Letzteres gilt auch dann, wenn eine Versorgungsleitlinie im obigen Sinne vorläge. Die Bindung ist eine Folge des Professionsvorbehalts und der Seriositätsindizien der hochwertigen Leitlinie, die gerade für die anderen der G-BA-Entscheidung zugrunde liegenden Kriterien nicht in Anspruch genommen werden können. ${ }^{82}$ Diese Konsequenz wird in der einschlägigen sozialrechtlichen Literatur bisher nicht gezogen. ${ }^{83}$

77 Streitig; anders Clemens, Leitlinien und Sozialrecht, S. $147 \mathrm{ff} ., 160 \mathrm{f}$.

78 Francke, Leitlinien und Sozialrecht, S. 123 ff., bes. 141.

79 Francke, Leitlinien und Sozialrecht, S. 123 ff., bes. 143.

80 Clemens, Leitlinien und Sozialrecht - Rezeption von Leitlinien durch Rechtssetzung und Rechtsprechung, S. 147 ff., bes. 157; ebenso Francke, Leitlinien und Sozialrecht, S. 123 ff., bes. $143 \mathrm{f}$.

81 Ein Berücksichtigungsgebot und dann wohl auch Bindung für S3-Leitlinien begründet Francke, Leitlinien und Sozialrecht, S. 123 ff., bes. $128 \mathrm{ff}$.

82 Hart, Qualitätssicherung durch Leitlinien, VSSR 2002, S. 265-297, bes. 281, 283 f.

83 Clemens, Leitlinien und Sozialrecht S. 147 ff., bes. 156 ff.; Francke, Leitlinien und Sozialrecht, S. 123 ff., bes. $143 \mathrm{f}$. 
Die Zweckmäßigkeitsbindung (= hochwertige Leitlinie bestimmt Zweckmäßigkeit der Krankenversorgung in der GKV) zieht den Rahmen für die allgemeine und abstrakte Wirtschaftlichkeitsentscheidung:

$>$ Bei gleichermaßen zweckmäßigen Behandlungen ${ }^{84}$ ist die effizientere, also kostengünstigere vorzuziehen (das gilt ebenso für die vertragsärztliche Entscheidung).

$>$ Existiert nur eine einzige Behandlung, spielen Wirtschaftlichkeitsüberlegungen bei der »Zulassungsentscheidung « keine Rolle, es sei denn, es wäre ein Ausschlussoder ein Rationierungstatbestand erfüllt. ${ }^{85}$

$>$ Existieren mehrere unterschiedlich zweckmäßige (Differenzierungen hinsichtlich des Nutzens oder der Risiken ${ }^{86}$ ) Behandlungen und präferiert die hochwertige Leitlinie eine von ihnen und will der G-BA bei unterschiedlicher Effizienz eine nicht leitlinienempfohlene Behandlung aus Wirtschaftlichkeitsgründen als einzige »zulassen«, so kann er das wegen der Zweckmäßigkeitsbindung nur aufgrund einer die explizite Rationierung legitimierenden Norm. Das SGB V enthält bisher keine solche Rationierungsnorm. ${ }^{87}$

Faktisch muss man deshalb von einer relativ intensiven regulatorischen Wirkung der hochwertigen Leitlinie auch im Bereich der Effizienzregulation ausgehen.

\section{Leitlinie bestimmt Wirtschaftlichkeit?}

Folgt man den vorangehenden Ausführungen (II, III), so können sich hinsichtlich der sozialrechtlichen Entscheidungen jenseits des Kriteriums der Zweckmäßigkeit auch durch Versorgungsleitlinien keine Bindungswirkungen, sondern bestenfalls Berücksichtigungswirkungen ergeben. ${ }^{88}$ Dasselbe gilt für Leitlinien, die im Nationalen Programm für Versorgungsleitlinien bei der B $\ddot{\mathrm{AK}}{ }^{89}$ entstehen. $^{90}$

84 Immer vorausgesetzt in derselben Indikation.

85 Dazu Francke, Begrenzung der Leistungen der gesetzlichen Krankenversicherung - Grundund Wahlleistungen, Rationierung, Priorisierung, GesR 2003, 97 ff.; ders., Materielle Grenzen und rechtliche Verfahren der Festlegung eines GKV-Grundleistungskataloges, in: Norbert Klusen/Christoph Straub (Hrsg.), Bausteine für ein neues Gesundheitswesen Technik, Ethik, Ökonomie, 2003, S. 190 ff.; zu Leistungspflichten und Budgetierung Francke/Schnitzler, Die Behandlungspflicht des Vertragsarztes bei begrenzten Finanzmitteln Zur Unzulässigkeit der Verweigerung unrentabler Leistungen, SGb 2002, $84 \mathrm{ff}$.

86 Siehe dazu Hart, Der regulatorische Rahmen der Nutzenbewertung: Vom Arzneimittelrecht zum HTA-Recht, MedR 2004, 469 ff., bes. 475 ff.; zur Nutzen/Risiko-Abwägung im Arzneimittelrecht ders., Die Nutzen/Risiko-Abwägung im Arzneimittelrecht. Ein Element des Health Technology Assessment, Bundesgesundheitsbl - Gesundheitsforsch - Gesundheitsschutz $2005 \cdot 48: 204-214$.

$87 \S 92$ I S. 1, 3. HS SGB ${ }^{\circ}$ V verfolgt dieses Ziel wohl nicht; siehe Hart, Der regulatorische Rahmen der Nutzenbewertung, MedR 2004, 469 ff., 472; insgesamt Francke, Begrenzung der Leistungen der gesetzlichen, GesR 2003, $97 \mathrm{ff}$.

88 Ebenso Clemens, Leitlinien und Sozialrecht S. 147 ff., bes. 156 ff.; Francke, Leitlinien und Sozialrecht, S. 123 ff., bes. 139 f, 143 f.; Mengel, Sozialrechtliche Rezeption, S. 185 ff., 297 ff.

89 Nationales Programm für Versorgungsleitlinien bei der Bundesärztekammer (ZaeFQ (2002) 96: Suppl. II, 1-24); dazu Ollenschläger u. a., Qualität und Akzeptanz, S. 17 ff., bes. $31 \mathrm{ff}$.

90 Francke, Leitlinien und Sozialrecht, S. 123 ff., bes. 141 f. 
Schaubild 5

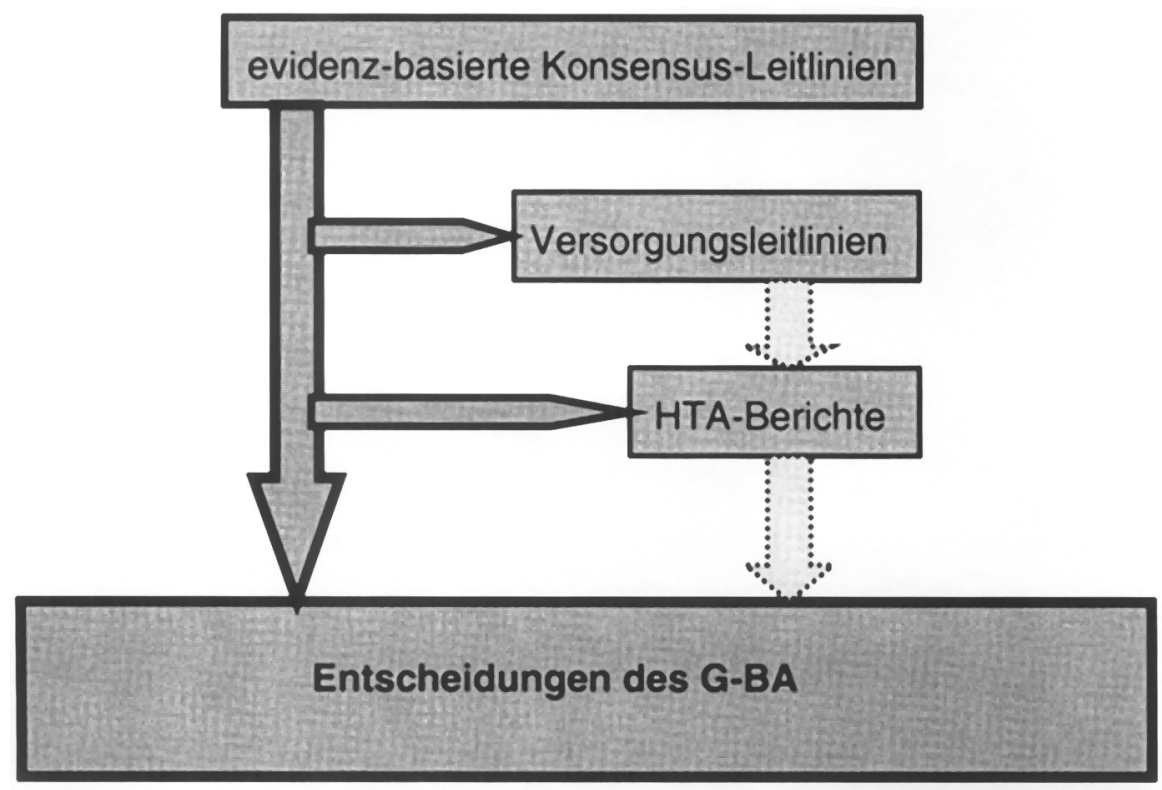

Hochwertige medizinische Leitlinien sind Versorgungsleitlinien und HTA-Berichten zugrunde zu legen, weil sie den Standard ordnungsgemäßer Behandlung festsetzen; Versorgungsleitlinien sind bei der Erstellung von HTA-Berichten zu berücksichtigen, können aber in keiner Weise binden. Die Verabredungsseriosität jenseits des Professionsvorbehalts ist entweder bisher nicht ausreichend entwickelt oder die erforderlichen Einlösungsanstrengungen werden nicht praktiziert. HTA-Berichten kommt eine die Entscheidungen des G-BA vorbereitende, nie eine bindende Funktion zu.

\section{Resümee}

Es erscheint folgerichtig, der »evidenz-basierten Konsensus-Leitlinie« die Funktion der medizinischen Standardfestsetzung zuzusprechen und daraus die medizin- wie gesundheitsrechtlichen Konsequenzen zu ziehen.

Medizinrechtlich bestimmt dieser Leitlinientypus den Standard und die Pflicht und die Abweichung von ihr die Pflichtverletzung (= Behandlungsfehler). Das Fehlen von Abweichungsgründen bzw. deren fehlende Dokumentation vermutet die haftungsbegründende Kausalität.

Gesundheitsrechtlich bestimmt dieser Leitlinientypus die Zweckmäßigkeit der Versorgung im GKV-Recht und gleichermaßen den Rahmen für die Entscheidung über die Wirtschaftlichkeit der Leistungserbringung. 